Check for updates

Cite this: RSC Adv., 2019, 9, 26572

\title{
Niclosamide encapsulated polymeric nanocarriers for targeted cancer therapy $\dagger$
}

\author{
Nishant Kumar Jain, ${ }^{a}$ Prabhuraj R. S., ${ }^{b}$ M. C. Bavya, ${ }^{a}$ Rajendra Prasad, (D) ${ }^{a}$ \\ Rajdip Bandyopadhyaya, (D) ${ }^{c}$ V. G. M. Naidu ${ }^{d}$ and Rohit Srivastava (DD *a
}

Localized cancer rates are on an upsurge, severely affecting mankind across the globe. Timely diagnosis and adopting appropriate treatment strategies could improve the quality of life significantly reducing the mortality and morbidity rates. Recently, nanotherapeutics has precipitously shown increased efficacy for controlling abnormal tissue growth in certain sites in the body, among which ligand functionalized nanoparticles (NP) have caught much attention for improved survival statistics via active targeting. Our focus was to repurpose the antihelminthic drug, niclosamide (NIC), which could aid in inhibiting the abnormal growth of cells restricted to a specific region. The work here presents a one-pot synthesis of niclosamide encapsulated, hyaluronic acid functionalized core-shell nanocarriers [(NIC-PLGA NP)HA] for active targeting of localized cancer. The synthesized nanocarriers were found to possess spherical morphology with mean size of $150.8 \pm 9 \mathrm{~nm}$ and zeta potential of $-24.9 \pm 7.21 \mathrm{mV}$. The encapsulation efficiency was found to be $79.19 \pm 0.16 \%$ with a loading efficiency of $7.19 \pm 0.01 \%$. The nanohybrids exhibited extreme cytocompatibility upon testing with MDA-MB-231 and L929 cell lines. The rate of cancer cell elimination was approximately $85 \%$ with targeted cell imaging results being highly convincing. [(NIC-PLGA NP)HA] demonstrates increased cellular uptake leading to a hike in reactive oxygen species (ROS) generation, combating tumour cells aiding in the localized treatment of cancer and associated therapy.

Received 6th May 2019

Accepted 14th August 2019

DOI: $10.1039 / c 9 r a 03407 b$

rsc.li/rsc-advances

\section{Introduction}

Cancer is defined as group of distinctive disorders characterized by the uncontrolled multiplication of cells. ${ }^{1}$ Cancer cells have an increased tendency to proliferate and spread to other body parts eventually displacing healthy cells leading to fatalities. ${ }^{2}$ Though attempts to destroy the abnormal cell division were implemented in late 40's nothing seemed successful., Numerous factors like metabolism, age, immune system, drugdrug interactions and several interpersonal variations are the foremost factors which contribute to this dreadful disorder., ${ }^{5,6}$ Considering this fact, there is no gold standard protocolized treatment to combat this life threatening disorder. ${ }^{7-9}$ To deal with this matter, the science of manipulating individual atoms and molecules, "Nanotechnology", has come forward with the

\footnotetext{
${ }^{a}$ Department of Biosciences and Bioengineering, Indian Institute of Technology Bombay (IIT-B), Powai, Mumbai, 400076, India. E-mail: rsrivasta@iitb.ac.in

${ }^{b}$ Centre for Research in Nanotechnology and Science, Indian Institute of Technology Bombay (IIT-B), Powai, Mumbai, 400076, India

${ }^{c}$ Department of Chemical Engineering, Indian Institute of Technology Bombay (IIT-B), Powai, Mumbai, 400076, India

${ }^{d}$ Department of Pharmacology \& Toxicology, National Institute of Pharmaceutical Education and Research (NIPER), Guwahati, Assam, 781125, India

$\dagger$ Electronic supplementary information (ESI) available. See DOI: 10.1039/c9ra03407b
}

design of drug carriers and various other systems to diagnose, cure and mitigate cancer. ${ }^{\mathbf{1 0 , 1 1}}$ Nanotherapy is the novel mode of treatment strategy applied for curing cancer and there is a need to address various concerns in pharmacotherapy such as; the property of substance and its optimization, and safety issues at the nanolevel. ${ }^{12}$ An ideal nanocarrier to combat cancer should respond to stimuli ${ }^{\mathbf{1 3}}$ able to attach with imaging specimens, biomarkers, genetic material and should be inert with drugs presenting high encapsulation efficiency.,14 Even the nanocarriers suffer from various hurdles such as; sophisticated design, time-consuming process, high cost products, low product yield, poor cellular internalization and therapeutic ability if not synthesized vigilantly. ${ }^{15,16}$ Nanomedicine platforms were considered to be a therapeutic arsenal for cancer treatment with tremendous impact years ago with the advent of Doxil and Myocet, being clinically approved along with liposomal formulations. ${ }^{17}$ With regard to localized cancer therapy, controlled release of chemotherapeutics has paved the way for destruction of tumour development. ${ }^{18}$ In this work, we proposed the synthesis of niclosamide (NIC) loaded, poly-lactic-co-glycolic acid (PLGA) nanoparticles (NP) further functionalized with hyaluronic acid for effective targeting.

Our approach was in choosing FDA approved biomaterials for designing the nanoformulation so as to prevent complications of toxicity. Study here discusses the synthesis, 
characterizations and efficacy of NIC encapsulated PLGA nanocarrier functionalized with hyaluronic acid to combat against cancer. PLGA has been utilized in the study due to its commonness being used in prolonged clinical and biomedical applications. ${ }^{19}$ PLGA has good biocompatibility, degradability, and is an ample nanocarrier in exhibiting controlled drug delivery. ${ }^{20}$ Moreover, owing to flexibility in surface properties of PLGA, it could be tuned with respect to the biomedical applications. ${ }^{21}$ Moreover, PLGA has gained US-FDA approval to be used as a safe biomaterial. ${ }^{22,23}$ Despite merits, PLGA suffers from demerits like; poor colloidal stability, low product yield, uncontrolled particle morphology, low cargo capacity, fast aggregation, slow degradation, poor cellular uptake which are the major issues to be discussed requisitely. ${ }^{24}$ To overcome these limitations we have surface modified PLGA nanoparticles with hyaluronic acid to further promote active targeting. ${ }^{25}$ PLGA NPs were synthesized via emulsion solvent evaporation method and functionalized using hyaluronic acid. Hyaluronic acid (HA) is a linear mucopolysaccharide, which plays a vital role in extracellular matrix. HA is available in different molecular weights of which, higher molecular weight HA is preferred over others due to its anti-angiogenic, ${ }^{26}$ anti-inflammatory ${ }^{27}$ and high CD44 binding capacity. ${ }^{28}$ HA modification of nanocarriers has led to enhancement in drug delivery, to actively target CD44 overexpressed cancer cells and suppress them further impeding its progression. ${ }^{29,30}$

The drug of choice employed for the study was well known FDA approved anthelmintic drug, niclosamide. ${ }^{31}$ NIC is a teniacide with reported activities to be involved in inhibiting signaling pathways to serve against cancer progression, whereby creating a multi-purposing or repurposing of an existing drug. The drug used is cost effective that formulate an affordable formulation when compared to most of first choice of drugs used in cancer treatments. Moreover, the drug has shown promising anti-cancer activity against 60 different human cancer cell lines. ${ }^{32}$ The significant therapeutic action of NIC with regard to cancer therapy is in blocking of multiple intracellular signalling pathways like STAT3, Wnt/ $\beta$-catenin, mTORC1, NF- $\kappa \mathrm{B}$, and Notch. ${ }^{33-37}$ Therefore, an efficient drug delivery system that can deliver this drug to the target site needs to be developed. Therefore, our basic research strategy was in the developing cost effective, readily available biomaterials being synthesized in a facile one pot strategical approach to fight against cancer leading to complete tumour suppression and further hindering its progression to specific sites of the body.

\section{Materials and methods}

\subsection{Materials}

Polymer poly (lactic-co-glycolic) acid (PLGA) (50:50, $17 \mathrm{kDa}$ ) was obtained as gift sample from Corbion Purac Biomaterials (Gorinchem, Netherlands). Hyaluronic acid (MW 1000-1600 $\mathrm{kDa}$ ) was purchased from Kumar Organic Products Ltd. (Bangalore, India). Niclosamide and fluorescein isothiocyanate (FITC) were acquired from Sigma-Aldrich (St. Louis, USA). Polyvinyl alcohol (PVA) (85-125 kDa molecular weight, 86-89\% degree of hydrolysis) was purchased from S. D. Fine-Chem Limited (Mumbai, India). Organic solvents (HPLC grade); ethyl acetate, and acetone were bought from Merck Pvt., Ltd. (Mumbai, India). The MDA-MB-231 and L929 cells were procured from the cell repository at National Centre for Cell Science (NCCS), Pune, Maharashtra, India. Dulbecco's phosphate-buffered saline (DPBS), Dulbecco's modified Eagle's medium (DMEM), fetal bovine serum (FBS), streptomycin, trypsin and cellulose dialysis bag were purchased from Himedia (Mumbai, India). 3-(4,5-Dimethylthiazol-2-yl)-2,5diphenyltetrazolium bromide (MTT), 2',7'-dichlorofluorescin diacetate (DCFDA), propidium iodide and RNase A were purchased from Sigma-Aldrich (St. Louis, United States). All other chemicals used in the study were of analytical grade purchased from Merck Pvt., Ltd. (Mumbai, India) and were used as received without further alteration. Experiments were performed in triplicates for confirmation of results.

\subsection{Preparation of HA functionalized PLGA nanoparticles}

Niclosamide encapsulated PLGA nanoparticles (NIC-PLGA NP) were prepared by emulsion solvent evaporation method. ${ }^{38}$ Briefly, $25 \mathrm{mg}$ of PLGA and $2.5 \mathrm{mg}$ of NIC was dissolved in $5 \mathrm{~mL}$ of ethyl acetate to give organic phase. This organic solution was added dropwise into $10 \mathrm{~mL}$ of aqueous phase containing $1 \% \mathrm{w} / \mathrm{v}$ PVA under gentle stirring. Subsequently, the emulsion formed was sonicated for $10 \mathrm{~min}$ at $40 \%$ amplitude $(3 \mathrm{~s}$ on and $2 \mathrm{~s}$ off cycle). The emulsion obtained was subjected to magnetic stirring for 4-5 h assuring complete evaporation of ethyl acetate. The obtained dispersion was taken in a dialysis bag (MWCO 12 $\mathrm{kDa}$ ) and kept for dialysis in millipore water for $4 \mathrm{~h}$ to remove the unentrapped drug. The uniform dispersion of nanoparticles formed were stored at $4{ }^{\circ} \mathrm{C}$. The particles upon lyophilisation were utilized for further studies. Simultaneously, drug loaded PLGA NP with HA coating [(NIC-PLGA NP)HA] were prepared by using the same method, additionally incorporating $0.1 \% \mathrm{w} / \mathrm{v}$ $\mathrm{HA}$ in the aqueous phase. Bare PLGA NP and [(PLGA NP)HA] were also prepared for result comparison. On the other hand, FITC loaded PLGA nanoparticles FITC-PLGA NP and [(FITCPLGA NP)HA] were prepared by replacing drug with dye (with $10 \%$ loading ratio) to aid in carrying out cell uptake studies.

\subsection{Characterization of nanoparticles (NP)}

2.3.1 Hydrodynamic diameter, zeta potential, encapsulation and loading efficiency. Hydrodynamic diameter, polydispersity index (PDI) and zeta potential (surface charge, $\zeta$ ) of the developed NP were measured using Zetasizer Nano ZS 90 (Malvern Instruments Ltd., Worcestershire, UK). The mean hydrodynamic diameter ( $\mathrm{nm}$ ) of NP was measured by dynamic light scattering (DLS) at $25{ }^{\circ} \mathrm{C}$. The polydispersity index (PDI) was recorded for all samples to define the homogeneity of NP. The zeta potential of NP was measured using Laser Doppler Anemometry (LDA) to determine the electrophoretic mobility and particle stability. Nanoparticles were calculated for their encapsulation efficiency using direct method, where a weighed amount of drug loaded nanoparticles were dispersed in ethanol, followed by sonication for 5 min ( $40 \%$ amplitude) and kept in 
shaker for $24 \mathrm{~h}^{39-42}$ Subsequently, the nanoparticles were centrifuged at $15000 \mathrm{rpm}$ for $15 \mathrm{~min}$ at $4{ }^{\circ} \mathrm{C}$. Then the supernatant was collected and analysed for quantification of drug at $336 \mathrm{~nm}$ using UV spectrophotometer (Lambda 25, PerkinElmer). The percentage entrapment efficiency (EE) and loading efficiency (LE) were calculated using the following eqn (1) and (2): ${ }^{43}$

Entrapment efficiency $(\mathrm{EE}) \%$

$$
=\frac{\text { Total amount of drug in the nanoparticles } \times 100}{\text { Total amount of drug taken }}
$$

Loading efficiency $(\mathrm{LE}) \%$

$$
=\frac{\text { Total amount of drug in nanoparticle } \times 100}{\text { Yield of nanoparticle }}
$$

2.3.2 SEM imaging. Field Emission Gun-Scanning Electron Microscope (FEGSEM, JEOL JSM-7600 F) was used to observe the surface morphological characteristics of the prepared set of NP; PLGA NP, (NIC-PLGA NP), [(PLGA NP)HA] and [(NIC-PLGA $\mathrm{NP}) \mathrm{HA}$ ]. Approximately, $10 \mu \mathrm{L}$ of NP were drop-casted on the carbon tape placed over brass studs and were allowed to dry overnight at ambient temperature. Further, these dried samples were subjected to platinum sputter coating and measurements were recorded at an accelerating voltage of $10 \mathrm{kV}$.

2.3.3 Transmission electron microscopy (TEM). The transmission electron microscope (TEM, JEM 2100 JEOL Ltd., Tokyo, Japan) was used to observe the coating of HA onto the prepared [(NIC-PLGA NP)HA]. Around $10 \mu \mathrm{L}$ of sample was drop-casted on to copper grid coated with a thin film of carbon followed by air-drying the grid at room temperature. Subsequently, the samples were observed under a voltage of $200 \mathrm{kV}$. (NIC-PLGA NP) served as the control.

2.3.4 FTIR and XRD analysis. Nanoparticles PLGA NP, (NIC-PLGA NP), [(PLGA NP)HA] and [(NIC-PLGA NP)HA] were subjected to FTIR analysis using (3000 Hyperion Microscope with vertex 80 FTIR system). Spectrum recording was done from 4000 to $400 \mathrm{~cm}^{-1}$. Samples were pelletized by grinding with $\mathrm{KBr}$ of infrared grade and analyzed for the presence of functional groups. The peaks obtained were compared and contrasted with the individual compounds peak. X-ray diffraction studies of the nanoparticles were performed at $37{ }^{\circ} \mathrm{C}$ employing X-ray Rigaku Smart Lab powder X-ray diffractometer at $2 \theta$ ranging from 10 $80^{\circ}$ utilizing $\mathrm{K} \alpha$ copper radiation. The obtained data were plotted and analyzed for their phase identification. ${ }^{\mathbf{4 4}}$

2.3.5 Thermogravimetric analysis (TGA). Thermal stability of NIC, PLGA NP, (NIC-PLGA NP), and [(NIC-PLGA NP)HA] were analysed by Diamond TG/DTA, PerkinElmer, USA. The lyophilized nanoparticles $(\sim 2 \mathrm{mg})$ were placed in aluminium pans and thermograms were recorded under flow of nitrogen at a heating rate of $10{ }^{\circ} \mathrm{C} \min ^{-1}$ in the range from $25-500{ }^{\circ} \mathrm{C}$.

2.3.6 In vitro drug release. The in vitro release profiling of NIC from following formulations; (NIC-PLGA NP), and [(NICPLGA NP)HA] were performed in phosphate buffer saline (PBS) (pH 7.4). Briefly, $3 \mathrm{~mL}$ of the nanoparticulate suspension was transferred into a dialysis tube (MWCO $12000 \mathrm{Da})$ which was then placed into $12 \mathrm{~mL}$ of buffer medium containing $0.5 \%$ w/v Tween 80 . Further, the whole setup was placed in a shaker incubator maintained at a temperature of $37 \pm 2{ }^{\circ} \mathrm{C}$ at $100 \mathrm{rpm}$. Sample volume of $2 \mathrm{~mL}$ was withdrawn at predetermined time intervals followed by replacing the same amount with fresh buffer to maintain the sink condition. Quantification of the released drug was carried out using UV spectrophotometric analysis at a detection wavelength of $336 \mathrm{~nm}$ and calculated as per the following eqn (3) as follows:

Percentage niclosamide released $(\%)$

Amount of niclosamide released at particular interval of time $\times 100$

Amount of niclosamide encapsulated in the nanoparticle

\subsection{Cell culture studies}

2.4.1 Cytotoxicity assay. Cytotoxicity studies of the nanoparticulate formulations were performed on MDA-MB-231, metastatic triple negative breast cancer cells and L929, healthy mouse fibroblast cells. MTT assay was performed to evaluate the in vitro cytotoxicity of the prepared set of NP. Briefly, $100 \mu \mathrm{L}$ of cell suspension containing $5 \times 10^{3}$ cells per well were seeded in 96 well plate and allowed to incubate at $37{ }^{\circ} \mathrm{C}$ for $24 \mathrm{~h}$ in $5 \% \mathrm{CO}_{2}$ for cell attachment. Subsequently, the cells were treated with NIC, (NIC-PLGA NP), (PLGA NP), [(PLGA $\mathrm{NP}) \mathrm{HA}$ ] and [(NIC-PLGA NP)HA] containing an equivalent amount of drug ranging from 2 to $10 \mu \mathrm{M}$ and incubated at $37^{\circ} \mathrm{C}$ for $48 \mathrm{~h}$ in $5 \% \mathrm{CO}_{2}$. After $48 \mathrm{~h}$, the media was discarded and cells were washed with PBS. Further, $10 \%$ of MTT reagent was added to the cells and incubated for $4 \mathrm{~h}$ at $37{ }^{\circ} \mathrm{C}$ respectively. After which, media was removed, and $100 \mu \mathrm{L}$ of DMSO was added to dissolve the formazan crystal. Subsequently, the absorbance of the individual wells were recorded after incubation of $15 \mathrm{~min}$ under dark condition using Tecan Infinite M200 Pro microplate reader at $570 \mathrm{~nm}$ and background control at $620 \mathrm{~nm}$. The percentage cell viability was calculated using the following eqn (4). Similarly, biocompatibility of bare (PLGA NP) and [(PLGA NP)HA] were checked on MDA-MB-231 and L929 cells in varying concentration from 50 to $250 \mu \mathrm{g} \mathrm{mL}{ }^{-1}$.

$$
\text { Cell viability }(\%)=\frac{\text { Mean OD }(\text { sample })}{\text { Mean OD }(\text { negative control })} \times 100
$$

2.4.2 Confocal laser scanning microscopy. Confocal microscopy was performed to investigate the internalization and targeting ability of HA-coated PLGA NP towards the proteins on the cell membrane in MDA-MB-231 cells. In short, $1 \times 10^{5}$ cells per well were seeded in 12-well plates and incubated at $37{ }^{\circ} \mathrm{C}$ for $24 \mathrm{~h}$ in $5 \%$ $\mathrm{CO}_{2}$ to allow the attachment of cells on the sterile glass coverslip which was placed at the bottom of each well. FITC-PLGA NP and [(FITC-PLGA NP)HA] (equal to $20 \mu \mathrm{M}$ of niclosamide loaded nanoparticles) were added to cells and incubated for $12 \mathrm{~h}$ at $37^{\circ} \mathrm{C}$. The cells were then washed with $1 \mathrm{~mL}$ of PBS to remove the dead cells 
and remaining cells were fixed with $1 \mathrm{~mL}$ of $3.7 \%$ of formaldehyde for $15 \mathrm{~min}$. The formaldehyde solution was then removed from wells and each well was again washed with PBS. The imaging of cells was carried out under confocal laser scanning microscope (Zeiss Axio-Observer Z1 microscope).

2.4.3 Internalization, ROS and cell-cycle analysis using flow cytometry. Cells $\left(2 \times 10^{5}\right.$ cells per $\left.\mathrm{mL}\right)$ were seeded in T-25 flasks and allowed to reach $70-80 \%$ confluency. For internalization, FITC-PLGA NP and [(FITC-PLGA NP)HA] (equal to $20 \mu \mathrm{M}$ of NIC loaded nanoparticles) were added to cells and then incubated for $24 \mathrm{~h}$ at $37{ }^{\circ} \mathrm{C}$ in $5 \% \mathrm{CO}_{2}$. The cells were then washed with PBS and harvested after treatment with trypsin solution followed by centrifugation. The pellet obtained was redispersed in $500 \mu \mathrm{L}$ of PBS for internalization study using flow cytometer (BD FACS Aria, USA). Similarly, cells were treated with NIC, (NIC-PLGA NP) and [(NICPLGA NP)HA] containing drug equivalent of $20 \mu \mathrm{M}$ and incubated for $24 \mathrm{~h}$ for ROS and cell cycle analysis. Oxidative stress inside the MDA-MB-231 cells, a peroxidase sensitive fluorescent probe $2^{\prime}, 7^{\prime}$ dichlorodihydrofluorescein diacetate (DCFDA) was used due to its extreme sensitivity to track changes in redox state of cells over period of time. Cells were treated with DCFDA solution prepared in PBS $(2 \mu \mathrm{M})$ and kept in the incubator for $10 \mathrm{~min}$ at $37^{\circ} \mathrm{C}$. For cell cycle analysis, after completing the treatment period, the cells were washed with PBS, harvested with trypsin solution and fixed in $1 \mathrm{~mL}$ of $70 \%$ chilled ethanol. The fixed cells were then centrifuged for 5 min, discarding the ethanol. The pellet was further treated with $500 \mu \mathrm{L}$ of PBS containing PI $\left(50 \mu \mathrm{g} \mathrm{mL}{ }^{-1}\right)$ and RNase A $(100 \mu \mathrm{g}$ $\mathrm{mL}^{-1}$ ) and kept in dark for $15 \mathrm{~min}$. A minimum of 10000 events was recorded for each sample in flow cytometry and the results were analyzed using Flowjo V10.1R7 software.

\section{Results and discussion}

\subsection{Preparation of nanoparticles}

NIC drug loaded hyaluronic acid functionalized PLGA nanoparticles [(NIC-PLGA NP)HA] were synthesized using emulsion solvent evaporation method as shown in (Fig. 1). The functionalized coating of nanoparticles using HA was attained via physisorption upon sonicating the prepared nanodispersion under mild stirring. Coating emanated extreme targeting for localized cancer therapy exhibiting more negative surface charge with zeta potential of $-24.9 \pm 7.21 \mathrm{mV}$, when compared with the uncoated (NIC-PLGA NP) being $-11.2 \pm 5.02 \mathrm{mV}$ (ESI Fig. $\mathrm{S} 1 \dagger)$. It was noticed that the layer of HA has raised the

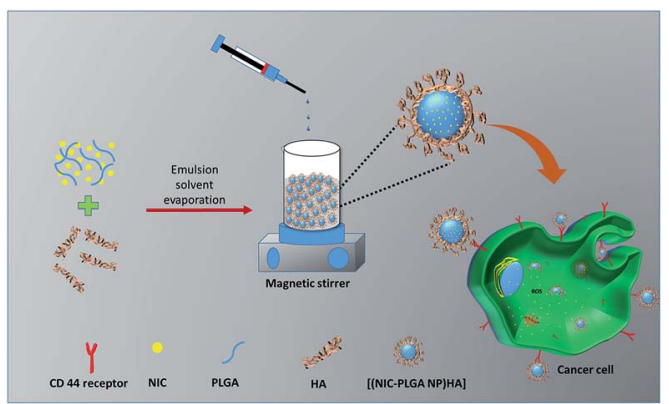

Fig. 1 Schematic representation showing synthesis and cellular uptake of [(NIC-PLGA NP)HA]. surface charge to more negative when compared to that of uncoated (NIC-PLGA NP), which is likely attributed with its negative surface charge. ${ }^{45}$ The prepared [(NIC-PLGA NP)HA] nanodispersion were found to exhibit a hydrodynamic diameter of $201.8 \pm 3.6 \mathrm{~nm}$ with a PDI value of 0.164 confirming the monodispersed of the prepared dispersion (ESI Fig. S2 $\dagger$ ). Further confirmation of size was performed using SEM imaging which revealed the spherical morphology of [(NIC-PLGA NP)HA] with a size $150.8 \pm 9.9 \mathrm{~nm}$ and that of (NIC-PLGA NP) to be 129.2 $\pm 10 \mathrm{~nm}$ (Fig. 2A and B). The subtle hike in the particle size of [(NIC-PLGA NP)HA] when compared with (PLGA NP) might occur due to drug encapsulation and that of (NIC-PLGA NP) due to surface functionalization which is seen in SEM imaging. Besides, TEM analysis ensured the functionalization with HA in [(NIC-PLGA NP)HA] (Fig. 2C). The synthesized nanoparticles were analyzed for their encapsulation efficiency which was found to be $79.19 \pm 0.16 \%$ and that of loading efficiency to be $7.19 \pm 0.01 \%$ (ESI Table S1†).

\subsection{FTIR and XRD analysis}

Functional groups present in the system were analyzed using FTIR analysis. The peaks seen at $1285 \mathrm{~cm}^{-1}, 1515 \mathrm{~cm}^{-1}$, $1613 \mathrm{~cm}^{-1}$ and $1650 \mathrm{~cm}^{-1}$ corresponds to the characteristic peaks of the drug niclosamide which is shown in (Fig. 3A). ${ }^{46}$ Peaks at $1740 \mathrm{~cm}^{-1}$ and $1750 \mathrm{~cm}^{-1}$ indicates the characteristic peak of bare PLGA NP which corresponds to the carbonyl group and the latter carbonyl ester bond as already reported in the literature. ${ }^{47}$ The peak widening of [(NIC-PLGA NP)HA] at $3420 \mathrm{~cm}^{-1}$ could be clearly defined which is due to drug incorporation into the nanocarrier. For hyaluronic acid the stretching frequency at $1640 \mathrm{~cm}^{-1}$ attributes to carbonyl group of HA. ${ }^{48}$ The same peak seen in the composite nanocarrier establishes the presence of hyaluronic acid functionalization in the system. The XRD pattern represents sharp peaks of NIC confirming its crystalline nature as shown in the (Fig. 3B). ${ }^{\mathbf{9}}$ While, the free nanoparticles shows relatively simple peaks. In [(NIC-PLGA NP)HA] formulation the drug peak is being moderately simple, which could be accounted due to the drug residing inside the molecular dispersion, which is affected by weak forces of attraction. This confirms the fact that the crystalline drug could alter its nature when present inside the nanodispersion.

\subsection{Thermogravimetric analysis}

The physical and chemical nature of the nanoparticulate formulation was measured as a function of temperature and plotted (Fig. 4A). The figure explains the thermal decomposition of [(NIC-PLGA NP)HA] to be initiated at $220^{\circ} \mathrm{C}$. While, that of the pure drug, niclosamide is seen to decompose at $230{ }^{\circ} \mathrm{C}$, where initial weight loss might be due to the release of water molecule. The subtle peak shift could be advocated due to the stronger and even molecular contact between the drug and functionalized nanocarrier. While, decomposition temperature of pure drug and (NIC-PLGA NP) are found to remain in the similar range. Therefore, evidences suggests the influence of HA has shifted the peaks due to stronger interactions, while 

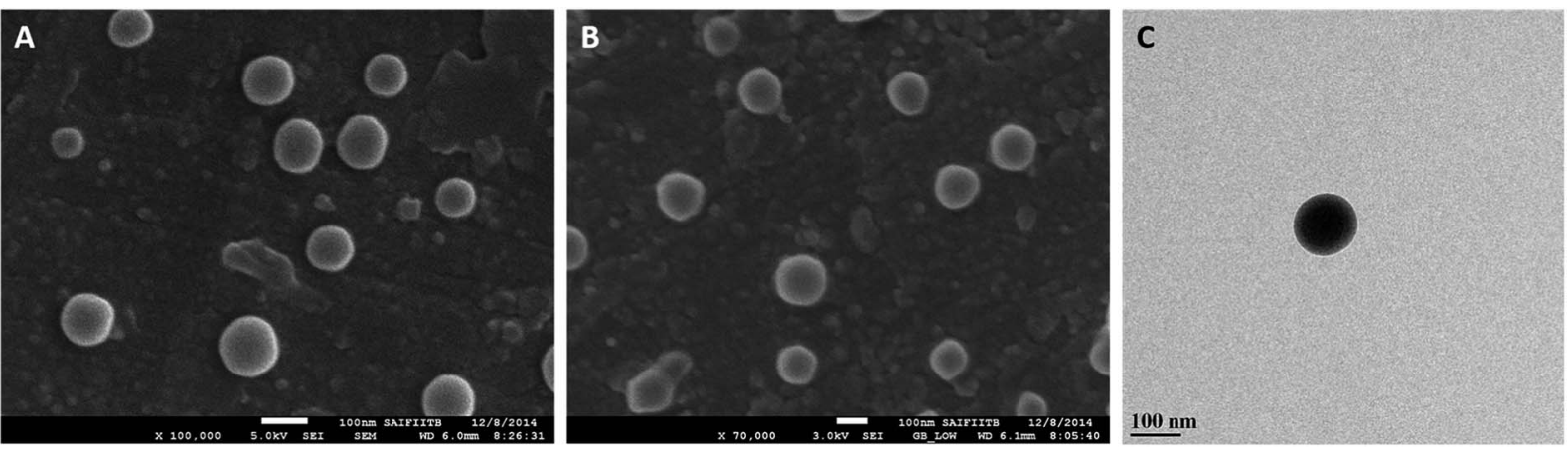

Fig. 2 SEM images of (A) NIC-PLGA NP and (B) [(NIC-PLGA NP)HA]; TEM image of (C) [(NIC-PLGA NP)HA].

without functionalization might be of less molecular interactions which may further affect the stability of the system. Functionalizing the drug loaded nanocarrier has reported to exhibit low rate of degradation with improved stability than the non-functionalized carrier molecule.

\subsection{Drug release}

The samples were assessed for drug release profiling and the release was calculated using UV spectrophotometer at a wavelength of $336 \mathrm{~nm}$. The release study was carried out in $\mathrm{pH} 7.4$ because the mean $\mathrm{pH}$ of extracellular tumor cells ranges from 5.7-7.8 and to show that it could also benefit as a prophylactic cancer treatment. Evidences states that niclosamide is seen to get released with an acidic $\mathrm{pH}$ trigger..$^{50-52}$ Drug release from the fabricated nanoparticles was carried in PBS at a $\mathrm{pH} 7.4$ containing $0.5 \% \mathrm{w} / \mathrm{v}$ of tween 80 as surfactant to maintain the sink condition (Fig. 4B). NIC release from (NIC-PLGA NP) is seemed to initially follow burst release further continued by constant pace release exhibiting $40.93 \pm 1.02 \%$ in $24 \mathrm{~h}$. Whereas, NIC from [(NIC-PLGA NP)HA] exhibited slow and sustained release compared to (NIC-PLGA NP) with a release percentage of $27.4 \pm$ $2.34 \%$ in $24 \mathrm{~h}$. The reason for the slow release could be attributed to the hydrophobic nature of the drug in addition; the release might also depend on the time taken to surpass the functionalized nanocarrier. NIC release from [(NIC-PLGA NP) $\mathrm{HA}]$ is found to completely release from the nanoparticulate formulation at $72 \mathrm{~h}$ with a release percentile of $55.53 \pm 2.37 \%$. The saturation in release might be due to decreased diffusion of the hydrophobic drug, which gets partitioned through the functionalized nanocarrier, possibly there had been some physical and chemical interactions occurring between the drugfunctionalized nanocarrier interfaces.

\subsection{Cytocompatibility assay}

The blank nanoparticles PLGA NP and [(PLGA NP)HA] didn't show any toxicity to both the MDA-MB-231 and L929 cells up to the maximum concentration of $250 \mu \mathrm{g} \mathrm{mL}{ }^{-1}$ which ensured that the nanoparticles are biocompatible (ESI Fig. S3†). The in vitro cytotoxicity of the prepared drug loaded functionalized polymeric nanoparticles on MDA-MB-231 for $48 \mathrm{~h}$ shown in Fig. 5A. Results indicated that the cancer cells when treated with [(NICPLGA NP)HA] showed significant cell death $(32 \%, 83 \%)$ at the concentration of 2 and $10 \mu \mathrm{M}$ respectively than (NIC-PLGA NP) $(27 \%, 73 \%)$ and NIC in DMSO $(21 \%, 72 \%)$. The targeted [(NICPLGA NP)HA] showed greater anticancer activity against cancer cells due to the potent targeting ability of HA compared to NICPLGA NP. Meanwhile, niclosamide in DMSO, (NIC-PLGA NP), [(NIC-PLGA NP)HA] exhibited similar cytotoxicity (cell viability $70 \%$ ) on healthy L929 cells, even at the highest concentration of $10 \mu \mathrm{M}$ (Fig. 5B). These results confirmed that niclosamide and [(NIC-PLGA NP)HA] were specific towards cancer cells than normal cells. The reason for [(NIC-PLGA NP)HA] was specific
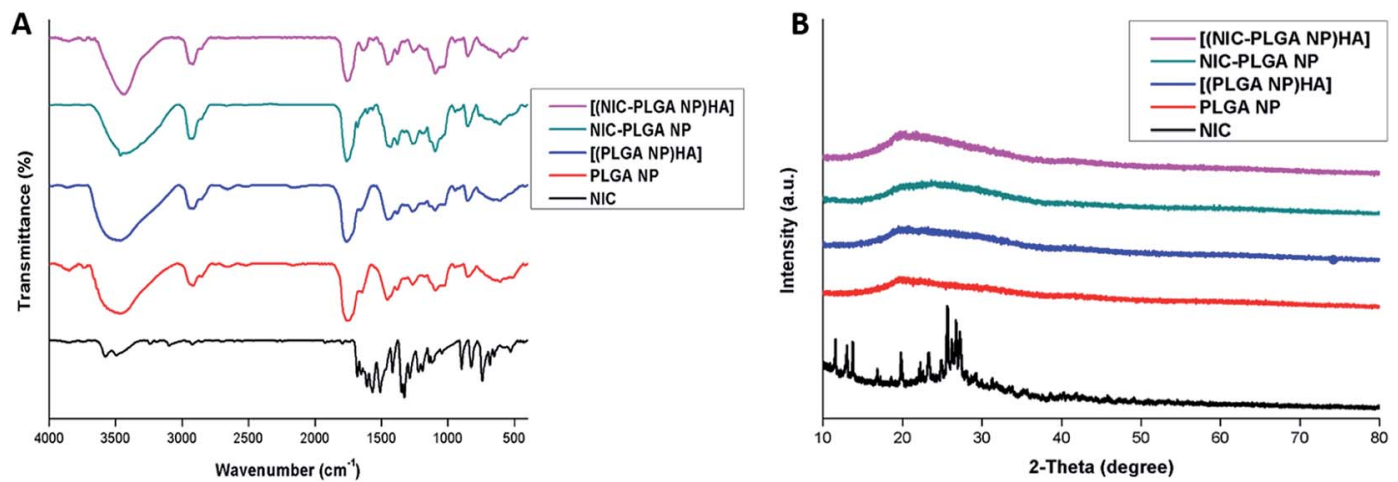

Fig. 3 (A) FTIR spectra of NIC, PLGA NP, [(PLGA NP)HA], NIC-PLGA NP and [(NIC-PLGA NP)HA]; (B) XRD pattern of NIC, PLGA NP, [(PLGA NP)HA], NIC-PLGA NP and [(NIC-PLGA NP)HA] 
A

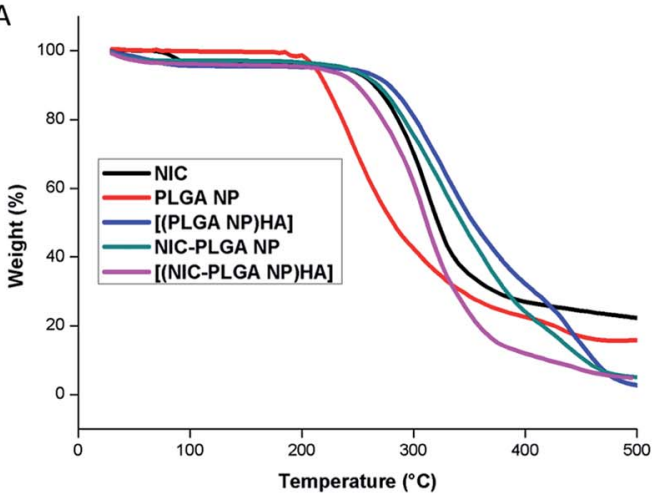

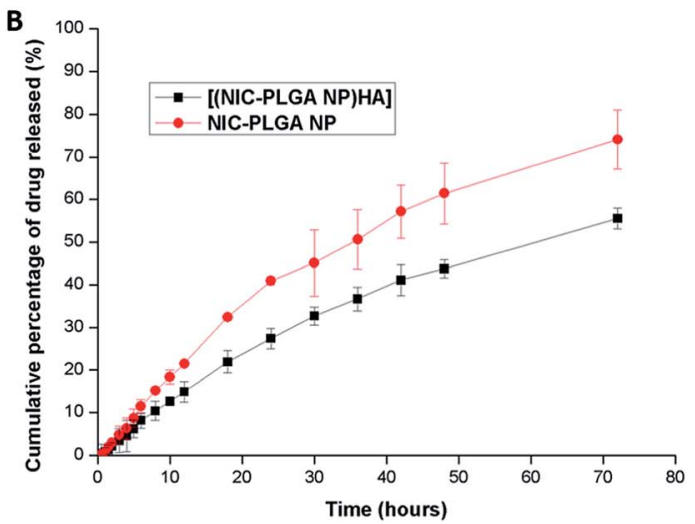

Fig. 4 (A) TGA thermogram of NIC, PLGA NP, [(PLGA NP)HA], NIC-PLGA NP and [(NIC-PLGA NP)HA]; (B) in vitro drug release study of NIC-PLGA $\mathrm{NP}$ and $[(\mathrm{NIC}-\mathrm{PLGA} \mathrm{NP}) \mathrm{HA}]$ in PBS $\mathrm{pH}$ 7.4. Data are expressed as mean $\pm \mathrm{SD}(n=3)$.

towards MDA-MB-231 cells can be attributed to overexpression of CD44 receptors on their surface.

\subsection{Cellular uptake and quantification studies}

The cellular uptake of nanoparticles in MDA-MB-231 breast cancer cell line was examined using confocal microscopy by loading FITC into PLGA NP. The (Fig. 6A and B) is found to exhibit green fluorescence only in the cell membrane of cells treated with [(FITC-PLGA NP)HA]. Whereas, green fluorescence was observed in cytoplasm of the cells treated with (FITC-PLGA $\mathrm{NP})$. It could be the fact that HA selectively binds to CD44 receptors overexpressed on the surface of MDA-MB-231 cells and are selectively uptaken through receptor-mediated endocytosis. ${ }^{\mathbf{4 2 , 4 3}}$ Previous literature reports have extensively compared different molecular weights of HA and concluded that high molecular weight HA exhibits increased CD44 receptor binding capacity than lower molecular weight HA. ${ }^{53}$ Likewise, the high molecular weight of HA used in the study supports in improving the CD44 receptor binding capacity. ${ }^{54}$ The quantification cellular internalization analysis performed on MDA-MB-231 cells revealed that [(FITC-PLGA NP)HA] showed more cellular uptake (79.28\%) compared to FITC-PLGA NP $(43.68 \%)$. The results obtained are in coordination with the recent literature. This might be advocated to the ability of HA in actively targeting cancer cells (Fig. 6C).

\subsection{Oxidative stress analysis}

ROS generation study was performed with DCFDA dye within the cancer cells, which leads to the apoptosis of cells. The production of ROS was found in the following order control (cells) < NIC < (NIC-PLGA NP) < [(NIC-PLGA NP)HA] (Fig. 6D). The amount of ROS produced by [(NIC-PLGA NP)HA] $(88.2 \%)$ was 4.36, 1.7 times higher than that obtained for NIC $(20.2 \%)$ and (NIC-PLGA NP) (51.9\%) respectively. Therefore it could be deduced that the disproportional rise in ROS produced by ([NICPLGA)HA] could induce cancer cell senescence and apoptosis. ${ }^{55}$ Apoptosis could be further related to hike in mitochondrial stress, releasing cytochrome $\mathrm{C}$ leading to irrevocable incidence whereby, activating the caspases resulting in complete cell death. ${ }^{56}$
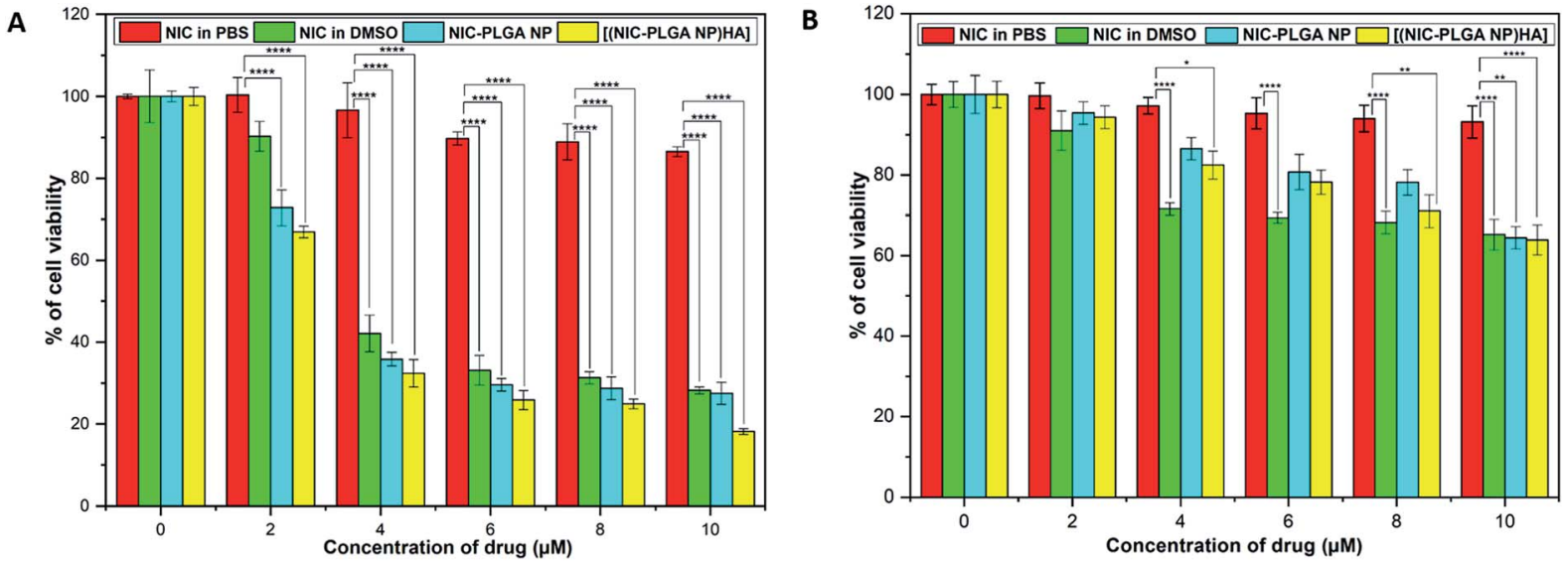

Fig. 5 In vitro cell viability analysis of NIC in PBS, NIC in DMSO, NIC-PLGA NP and [(NIC-PLGA NP)HA] in (A) MDA-MB-231 cells and (B) L929 cells. Each value represents the mean $\pm \mathrm{SD}(n=3)$. Statistical analysis was performed using one-way ANOVA, *P $\leq 0.05, * * P \leq 0.01, * * * P \leq 0.001$, $* * * * P \leq 0.0001$ 

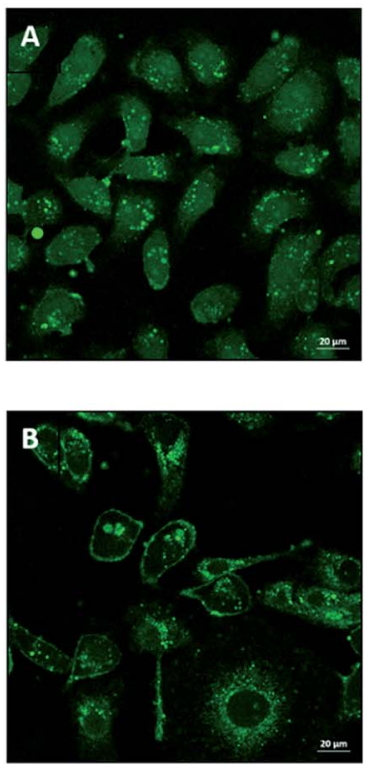
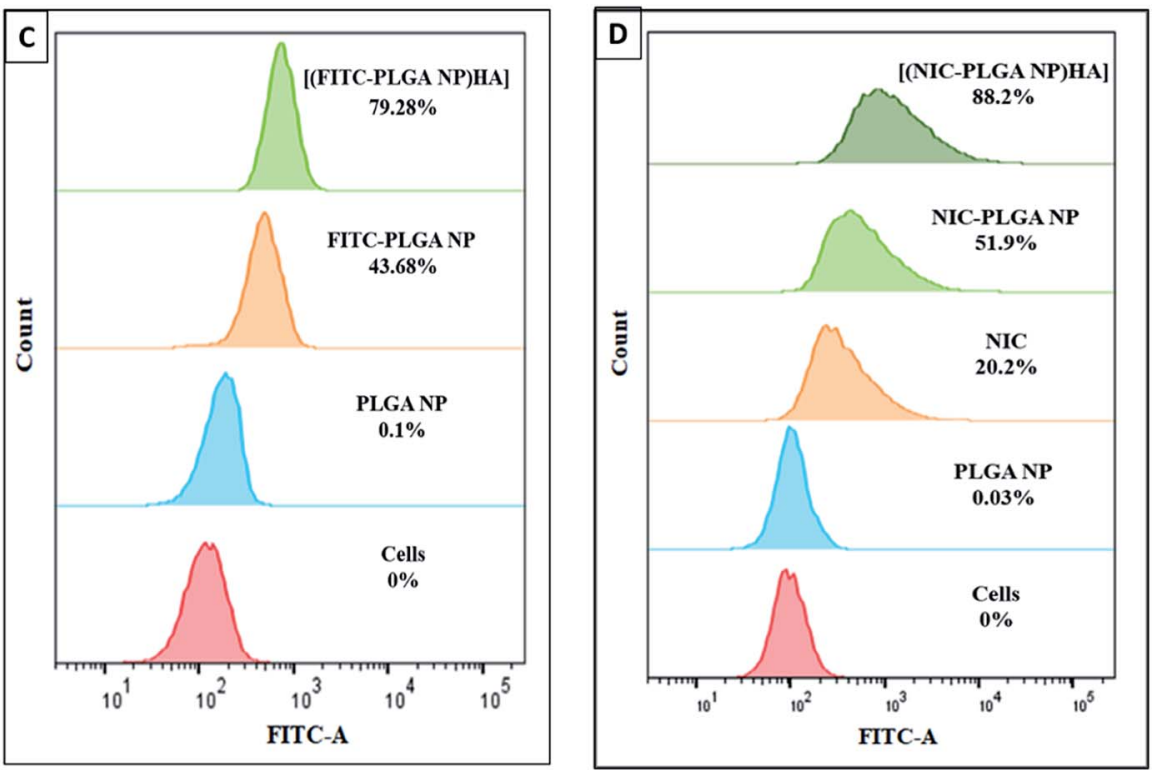

Fig. 6 Confocal microscopic images of cellular uptake of (A) FITC-PLGA NP and (B) [(FITC-PLGA NP)HA]; flow cytometry analysis of (C) cellular uptake of NIC, PLGA NP, FITC-PLGA NP and [(FITC-PLGA NP)HA] and (D) ROS generation study of NIC, PLGA NP, NIC-PLGA NP and [(NIC-PLGA NP)HA].

\subsection{Cell cycle analysis}

The results of cell cycle analysis reveals that the extent of dead cells which are exhibited in SubG1 phase, upon treatment with
PLGA NP was $0.28 \%$, affirming the compatibility of formulation (Fig. 7). On the other hand, SubG1 phase was found to increase significantly from $8.16 \%$ for NIC and $17.88 \%$ for (NIC-PLGA
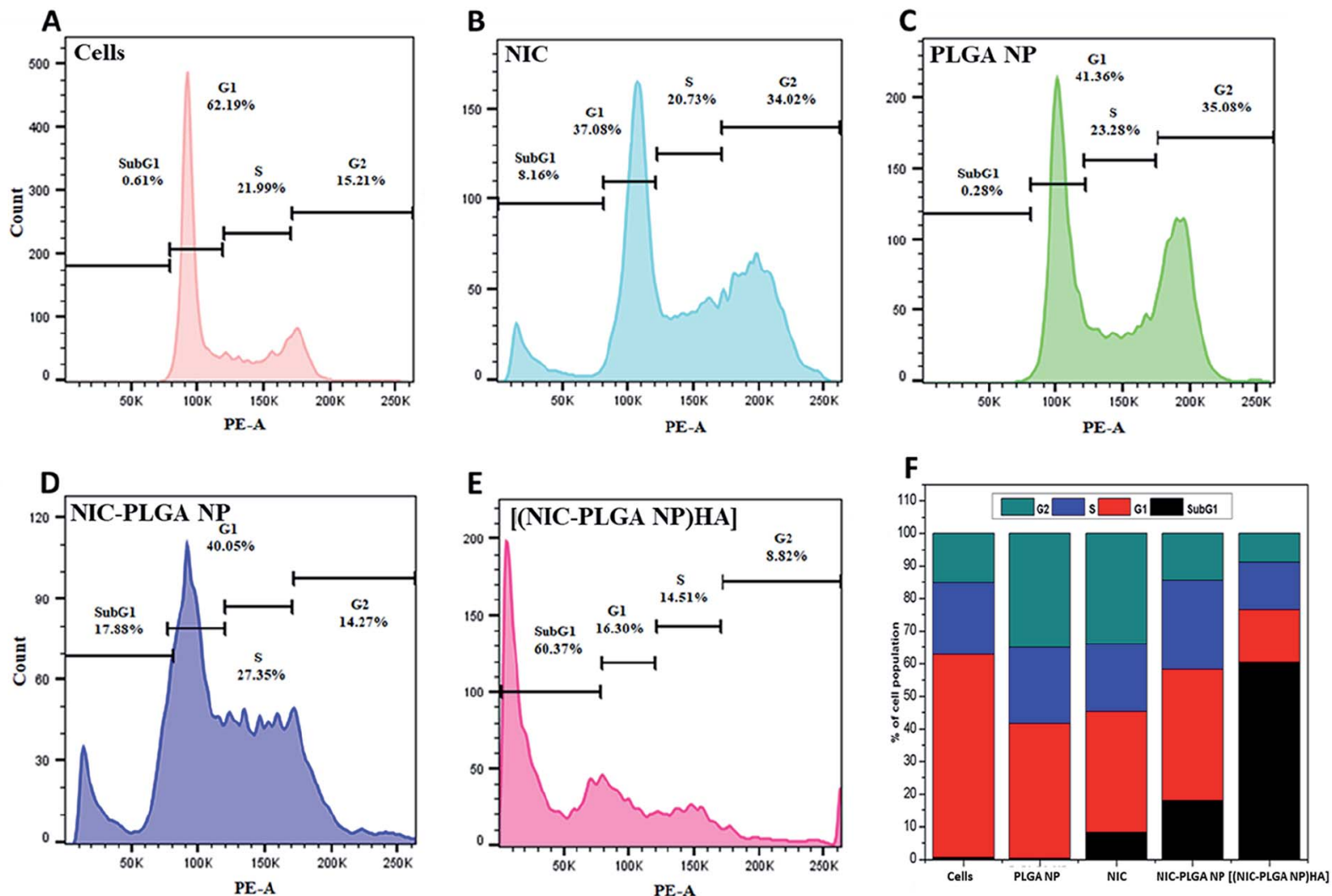

Fig. 7 Cell cycle analysis of (A) control cells, (B) NIC, (C) PLGA NP, (D) NIC-PLGA NP and (E) [(NIC-PLGA NP)HA]; and (F) comparative quantitative analysis of cell population in different phases of cell cycle. 
NP). Whereas, $60.37 \%$ of sudden rise in the Sub G1 Phase was observed in presence of [(NIC-PLGA NP)HA]. The observed increase in the Sub G1 phase of [(NIC-PLGA NP)HA] when compared to other formulations used in the study reveals the potential of its active targeting.

\section{Conclusions}

Contributions of nanotechnology in nano-biointeractions and personalization of nanomedicine in cancer cure has influenced mankind inexplicably. Inspite of the importance on extravasation in nanoparticle delivery, sustained drug delivery to localized abnormal cell growth and even permeation of nanotherapeutics on localized tumor growth remains pivotal in eliminating cancer. Niclosamide loaded PLGA nanoparticle were successfully synthesized via emulsion solvent evaporation method, which was further functionalized with hyaluronic acid for active targeting of cancer cells. [(NIC-PLGA NP)HA] exhibited improved stability with increased ROS production for combating cancer cell lines. The sustained release with HA functionalization proved to focus and kill cancer cells selectively. Biocompatibility studies on MDA-MB-231 and L929 cells proved to be remarkably convincing. Cellular uptake and quantification analysis results were consistent. However, more in vivo studies need to be carried out for acquiring knowledge regarding drug-drug interactions and associated side reactions, to promote formulation to clinical trials. To put in precise, [(NIC-PLGA NP)HA] nanoformulation could serve as active targeting agent for destroying abnormal cell division, and incorporation of dye in the formulation could help in cancer cell imaging.

\section{Conflicts of interest}

The authors declare no conflicts of interest for this work.

\section{Acknowledgements}

The authors are grateful to DST-INSPIRE, Department of Science and Technology, Government of India, New Delhi, India, for providing the fellowship during the research period. Authors would like to acknowledge DBT National Bioscience Award. The authors also thank SAIF-IIT Bombay (Mumbai, India) for providing the characterization facilities like SEM, TEM, FITR, XRD and TGA.

\section{References}

1 V. Wiwanitkit, Cancer nanotherapy: concept for design of new drug, J. Med. Hypotheses Ideas, 2013, 7, 3-4, DOI: 10.1016/j.jmhi.2012.10.002.

2 A. Ediriwickrema and W. M. Saltzman, Nanotherapy for Cancer: Targeting and Multifunctionality in the Future of Cancer Therapies, ACS Biomater. Sci. Eng., 2015, 1, 64-78, DOI: $10.1021 / \mathrm{ab} 500084 \mathrm{~g}$.

3 L. S. Goodman, M. M. Wintrobe, W. Dameshek, M. J. Goodman, A. Gilman and M. T. McLennan, Nitrogen mustard therapy: use of methyl-bis(beta-chloroethyl)amine hydrochloride and tris(beta-chloroethyl)amine hydrochloride for hodgkin's disease, lymphosarcoma, leukemia and certain allied and miscellaneous disorders, $J$. Am. Med. Assoc., 1946, 132, 126-132, DOI: 10.1001/ jama.1946.02870380008004.

4 S. M. Elbashir, J. Harborth, W. Lendeckel, A. Yalcin, K. Weber and T. Tuschl, Duplexes of 21-nucleotide RNAs mediate RNA interference in cultured mammalian cells, Nature, 2001, 411, 494, DOI: 10.1038/35078107.

5 B. Minke, C.-F. Wu and W. Pak, Continuous cultures of fused cells secreting antibody of predefined specificity, Nature, 1975, 254, 84-87, DOI: 10.1038/253600a0.

6 G. Rizzoli, L. Gregio, A. Mazzucco, P. Stritoni, A. Fracasso, T. Brumana and V. Gallucci, Determinants of late survival of 105 patients operated for dissection of the aorta, Eur. J. Cardiothorac. Surg., 1988, 2, 18-24, DOI: 10.1016/10107940(88)90091-7.

7 D. Peer, J. M. Karp, S. Hong, O. C. Farokhzad, R. Margalit and R. Langer, Nanocarriers as an emerging platform for cancer therapy, Nat. Nanotechnol., 2007, 2, 751-760, DOI: 10.1038/ nnano.2007.387.

8 A. K. Biswas, M. R. Islam, Z. S. Choudhury, A. Mostafa and M. F. Kadir, Nanotechnology based approaches in cancer therapeutics, Adv. Nat. Sci. Nanosci. Nanotechnol., 2014, 5, 043001, DOI: 10.1088/2043-6262/5/4/043001.

9 D. F. Costa, L. P. Mendes and V. P. Torchilin, The effect of low- and high-penetration light on localized cancer therapy, Adv. Drug Deliv. Rev., 2018, 138, 105-116, DOI: 10.1016/j.addr.2018.09.004.

$10 \mathrm{~J}$. Kreuter, Nanoparticles-a historical perspective, Int. J. Pharm., 2007, 331, 1-10, DOI: 10.1016/ j.ijpharm.2006.10.021.

11 A. Singh, M. Talekar, T.-H. Tran, A. Samanta, R. Sundaram and M. Amiji, Combinatorial approach in the design of multifunctional polymeric nano-delivery systems for cancer therapy, J. Mater. Chem. B, 2014, 2, 8069-8084, DOI: 10.1039/C4TB01083C.

$12 \mathrm{~V}$. Wiwanitkit, Advanced nanomedicine and nanobiotechnology, 2008.

13 R. Prasad, A. S. Yadav, M. Gorain, D. S. Chauhan, G. C. Kundu, R. Srivastava and K. Selvaraj, Graphene Oxide Supported Liposomes as Red Emissive Theranostics for Phototriggered Tissue Visualization and Tumor Regression, ACS Appl. Bio Mater., 2019, 2, 3312-3320, DOI: 10.1021/acsabm.9b00335.

14 Y. Opoku-Damoah, R. Wang, J. Zhou and Y. Ding, Versatile nanosystem-based cancer theranostics: design inspiration and predetermined routing, Theranostics, 2016, 6, 9861003, DOI: $10.7150 /$ thno.14860.

15 R. Huang, J. Vider, I. Serganova, R. G. Blasberg, M. Sloan, K. Cancer, M. Sloan and K. Cancer, Cancer nanomedicine: progress, challenges and opportunities, Nat. Rev. Cancer, 2014, 10, 215-226, DOI: 10.1038/nrc.2016.108.Cancer.

16 D. S. Anderson, M. J. Sydor, P. Fletcher and A. Holian, Nanotechnology: The Risks and Benefits for Medical 
Diagnosis and Treatment, J. Nanomed. Nanotechnol., 2016, 7, e143, DOI: 10.4172/2157-7439.1000.

17 Y. Barenholz, Doxil@ - the first FDA-approved nano-drug: lessons learned, J. Controlled Release, 2012, 160, 117-134, DOI: 10.1016/j.jconrel.2012.03.020.

18 A. Sneider, D. VanDyke, S. Paliwal and P. Rai, Remotely Triggered Nano-Theranostics For Cancer Applications, Nanotheranostics, 2017, 1, 1-22, DOI: 10.7150/ntno.17109.

19 H. K. Makadia and S. J. Siegel, Poly Lactic-co-Glycolic Acid (PLGA) as Biodegradable Controlled Drug Delivery Carrier, Polymers, 2011, 3, 1377-1397, DOI: 10.3390/ polym3031377.Poly.

20 F. Danhier, E. Ansorena, J. M. Silva, R. Coco, A. Le Breton and V. Préat, PLGA-based nanoparticles: an overview of biomedical applications, J. Controlled Release, 2012, 161, 505-522, DOI: 10.1016/j.jconrel.2012.01.043.

21 R. A. Jain, The manufacturing techniques of various drug loaded biodegradable poly(lactide-co-glycolide) (PLGA) devices, Biomaterials, 2000, 21, 2475-2490, DOI: 10.1016/ S0142-9612(00)00115-0.

22 J.-M. Lu, X. Wang, C. Marin-Muller, H. Wang, P. H. Lin, Q. Yao and C. Chen, Current advances in research and clinical applications of PLGA-based nanotechnology, Expert Rev. Mol. Med., 2009, 9, 325-341, DOI: 10.1586/ erm.09.15.Current.

23 J. S. Choi, K. Seo and J. W. Yoo, Recent advances in PLGA particulate systems for drug delivery, J. Pharm. Invest., 2012, 42, 155-163, DOI: 10.1007/s40005-012-0024-5.

24 N. Passerini and D. Q. M. Craig, An investigation into the effects of residual water on the glass transition temperature of polylactide microspheres using modulated temperature DSC, J. Control. Release., 2001, 73, 111-115, DOI: 10.1016/S0168-3659(01)00245-0.

25 G. Mattheolabakis, L. Milane, A. Singh and M. M. Amiji, Hyaluronic acid targeting of CD44 for cancer therapy: from receptor biology to nanomedicine, J. Drug Targeting, 2015, 23, 605-618, DOI: 10.3109/1061186X.2015.1052072.

26 R. Deed, P. Rooney, P. Kumar, J. D. Norton, J. Smith, A. J. Freemont and S. Kumar, Early-response gene signalling is induced by angiogenic oligosaccharides of hyaluronan in endothelial cells. Inhibition by nonangiogenic, high- molecular-weight hyaluronan, Int. J. Cancer, 1997, 71, 251-256, DOI: 10.1002/(SICI)10970215(19970410)71:2<251::AID-IJC21>3.0.CO;2-J.

27 P. L. Bollyky, B. A. Falk, R. P. Wu, J. H. Buckner, T. N. Wight and G. T. Nepom, Intact extracellular matrix and the maintenance of immune tolerance: high molecular weight hyaluronan promotes persistence of induced CD4+CD25+ regulatory T cells, J. Leukocyte Biol., 2009, 86, 567-572, DOI: $10.1189 / \mathrm{jlb} .0109001$.

28 P. M. Wolny, S. Banerji, C. Gounou, A. R. Brisson, A. J. Day, D. G. Jackson and R. P. Richter, Analysis of CD44-hyaluronan interactions in an artificial membrane system: insights into the distinct binding properties of high and low molecular weight hyaluronan, J. Biol. Chem., 2010, 285, 30170-30180, DOI: $10.1074 /$ jbc.M110.137562.
29 G. Huang and H. Huang, Application of hyaluronic acid as carriers in drug delivery, Drug Deliv., 2018, 25, 766-772, DOI: 10.1080/10717544.2018.1450910.

30 J. Kim, M. Moon, D. Kim, S. Heo and Y. Jeong, Hyaluronic Acid-Based Nanomaterials for Cancer Therapy, Polymers, 2018, 10, 1133, DOI: 10.3390/polym10101133.

31 H. Tao, Y. Zhang, X. Zeng, G. I. Shulman and S. Jin, Niclosamide ethanolamine-induced mild mitochondrial uncoupling improves diabetic symptoms in mice, Nat. Med., 2014, 20, 1263-1269, DOI: 10.1038/nm.3699.

32 Y. Li, P. K. Li, M. J. Roberts, R. C. Arend, R. S. Samant and D. J. Buchsbaum, Multi-targeted therapy of cancer by niclosamide: a new application for an old drug, Cancer Lett., 2014, 349, 8-14, DOI: 10.1016/j.canlet.2014.04.003.

33 K. Shuai, C. M. Horvath, L. H. T. Huang, S. A. Qureshi, D. Cowburn and J. E. Darnell, Interferon activation of the transcription factor Stat91 involves dimerization through SH2-phosphotyrosyl peptide interactions, Cell, 1994, 76, 821-828, DOI: 10.1016/0092-8674(94)90357-3.

$34 \mathrm{H}$. Clevers and R. Nusse, Wnt/ $\beta$-catenin signaling and disease, Cell, 2012, 149, 1192-1205, DOI: 10.1016/ j.cell.2012.05.012.

35 D. A. Guertin and D. M. Sabatini, Defining the Role of mTOR in Cancer, Cancer Cell, 2007, 12, 9-22, DOI: 10.1016/ j.ccr.2007.05.008.

36 W. E. Naugler and M. Karin, NF- $\kappa$ B and cancer - identifying targets and mechanisms, Curr. Opin. Genet. Dev., 2008, 18, 19-26, DOI: 10.1016/j.gde.2008.01.020.

37 I. M. Shih and T. L. Wang, Notch signaling, gamma-secretase inhibitors, and cancer therapy, Cancer Res., 2007, 67, 18791882, DOI: 10.1158/0008-5472.CAN-06-3958.

38 M. J. Ramalho and M. C. Pereira, Preparation and Characterization of Polymeric Nanoparticles: An Interdisciplinary Experiment, J. Chem. Educ., 2016, 93, 1446-1451, DOI: 10.1021/acs.jchemed.5b00837.

39 S. Sun, P. Liu, F. Shao and Q. Miao, Formulation and evaluation of PLGA nanoparticles loaded capecitabine for prostate cancer, Int. J. Clin. Exp. Med., 2015, 8, 19670-19681.

40 B. Gajra, C. Dalwadi and R. Patel, Formulation and optimization of itraconazole polymeric lipid hybrid nanoparticles (Lipomer) using box behnken design, Daru, J. Pharm. Sci., 2015, 23, 1-15, DOI: 10.1186/s40199-0140087-0.

41 K. S. Yadav and K. K. Sawant, Modified Nanoprecipitation Method for Preparation of Cytarabine-Loaded PLGA Nanoparticles, AAPS PharmSciTech, 2010, 11, 1456-1465, DOI: 10.1208/s12249-010-9519-4.

42 M. C. Pereira, L. E. Hill, R. Carlos, S. Mertens-talcott, S. Talcott and C. L. Gomes, LWT - Food Science and Technology Nanoencapsulation of hydrophobic phytochemicals using poly(DL-lactide-co-glycolide)(PLGA) for antioxidant and antimicrobial delivery applications: guabiroba fruit (Campomanesia xanthocarpa O. Berg) study, LWT-Food Sci. Technol., 2015, 63, 100-107, DOI: 10.1016/ j.lwt.2015.03.062.

43 M. C. Bavya, K. Vimal Rohan, G. B. Gaurav and R. Srivasatava, Synergistic treatment strategies to combat 
resistant bacterial infections using Schiff base modified nanoparticulate - hydrogel system, Mater. Sci. Eng. C, 2018, 95, 226-235, DOI: 10.1016/j.msec.2018.10.080.

44 L. George, M. C. Bavya, K. V. Rohan and R. Srivastava, A therapeutic polyelectrolyte-vitamin $\mathrm{C}$ nanoparticulate system in polyvinyl alcohol-alginate hydrogel: an approach to treat skin and soft tissue infections caused by Staphylococcus aureus, Colloids Surf., B, 2017, 160, 315324, DOI: 10.1016/j.colsurfb.2017.09.030.

45 L. Liu, F. Cao, X. Liu, H. Wang, C. Zhang, H. Sun, C. Wang, X. Leng, C. Song, D. Kong and G. Ma, Hyaluronic AcidModified Cationic Lipid-PLGA Hybrid Nanoparticles as a Nanovaccine Induce Robust Humoral and Cellular Immune Responses, ACS Appl. Mater. Interfaces, 2016, 8, 11969-11979, DOI: 10.1021/acsami.6b01135.

46 B. Bhushan, P. Dubey, S. U. Kumar, A. Sachdev, I. Matai and P. Gopinath, Bionanotherapeutics: niclosamide encapsulated albumin nanoparticles as a novel drug delivery system for cancer therapy, RSC Adv., 2015, 5, 12078-12086, DOI: 10.1039/C4RA15233F.

47 L. N. Okassa, H. Marchais, L. Douziech-Eyrolles, K. Hervé, S. Cohen-Jonathan, E. Munnier, M. Soucé, C. Linassier, P. Dubois and I. Chourpa, Optimization of iron oxide nanoparticles encapsulation within poly(d,l-lactide-coglycolide) sub-micron particles, Eur. J. Pharm. Biopharm., 2007, 67, 31-38, DOI: 10.1016/j.ejpb.2006.12.020.

48 M. Y. Shen, C. F. Chao, Y. J. Wu, Y. H. Wu, C. P. Huang and Y. K. Li, A design for fast and effective screening of hyaluronidase inhibitor using gold nanoparticles, Sens. Actuators B Chem., 2013, 181, 605-610, DOI: 10.1016/ j.snb.2013.02.054.

49 E. C. Van Tonder, T. S. P. Maleka, W. Liebenberg, M. Song, D. E. Wurster and M. M. De Villiers, Preparation and physicochemical properties of niclosamide anhydrate and two monohydrates, Int. J. Pharm., 2004, 269, 417-432, DOI: 10.1016/j.ijpharm.2003.09.035.

50 R. Martínez-Zaguilán, E. A. Seftor, R. E. B. Seftor, Y. W. Chu, R. J. Gillies and M. J. C. Hendrix, Acidic pH enhances the invasive behavior of human melanoma cells, Clin. Exp. Metastasis, 1996, 14, 176-186, DOI: 10.1007/BF00121214.

51 L. Xu, D. Fukumura and R. K. Jain, Acidic extracellular pH induces vascular endothelial growth factor (VEGF) in human glioblastoma cells via ERK1/2 MAPK signaling pathway. Mechanism of low pH-induced VEGF, J. Biol. Chem., 2002, 277, 11368-11374, DOI: 10.1074/ jbc.M108347200.

52 Y. Shen, H. Tang, M. Radosz, E. Van Kirk and W. J. Murdoch, $\mathrm{PH}-$ responsive nanoparticles for cancer drug delivery, Methods Mol. Biol., 2008, 437, 183-216, DOI: 10.1007/978-159745-210-6_10.

53 H. S. S. Qhattal and X. Liu, Characterization of CD44mediated cancer cell uptake and intracellular distribution of hyaluronan-grafted liposomes, Mol. Pharm., 2011, 8, 1233-1246, DOI: 10.1021/mp2000428.

54 S. Amorim, D. Soares, D. Freitas, C. A. Reis, R. L. Reis, I. Pashkuleva and R. A. Pires, Molecular weight of surface immobilized hyaluronic acid influences CD44-mediated binding of gastric cancer cells, Sci. Rep., 2018, 1-11, DOI: 10.1038/s41598-018-34445-0.

55 E. Cadenas, Mitochondrial free radical production and cell signaling, Mol. Asp. Med., 2004, 25, 17-26, DOI: 10.1016/ j.mam.2004.02.005.

56 H.-U. Simon, A. Haj-Yehia and F. Levi-Schaffer, Role of reactive oxygen species (ROS) in apoptosis induction, Apoptosis, 2000, 5, 415-418, DOI: 10.1023/A:1009616228304. 